\title{
Does Rapid Drug Desensitization to Chemotherapy Affect Survival Outcomes?
}

\author{
Berges-Gimeno MP ${ }^{*}$, Carpio-Escalona LV*, Longo-Muñoz F², Bernal-Rubio L', Lopez-Gonzalez P1, \\ Gehlhaar $\mathrm{P}^{1}$, Pachon $\mathrm{V}^{2}$, Ferreiro-Monteagudo $\mathrm{R}^{2}$, Madrigal-Burgaleta $\mathrm{R}^{1 * *}$, Alvarez-Cuesta $\mathrm{E}^{1 * *}$
}

${ }^{1}$ Allergy Division, Ramon y Cajal University Hospital, Madrid, Spain

${ }^{2}$ Medical Oncology Department, Ramon y Cajal University Hospital, Madrid, Spain

*These authors share first-author credit

**These authors share last-author credit

J Investig Allergol Clin Immunol 2020; Vol. 30(4): 254-263

doi: 10.18176/jiaci.0425

\begin{abstract}
Background: Hypersensitivity reactions to oxaliplatin may affect prognosis by jeopardizing the timely completion of scheduled treatment sessions or by forcing reactive patients into unexpected changes in therapy. Rapid drug desensitization (RDD) enables these patients to receive their first-choice treatments safely. However, the possible effects of RDD on the efficacy of oxaliplatin have never been studied. Objective: The objective of this study was to evaluate the effect of RDD on survival rates in oxaliplatin-hypersensitive patients. Methods: We performed a 7-year retrospective study to compare survival between oxaliplatin-hypersensitive cases (patients receiving oxaliplatin by RDD) and nonallergic controls (patients receiving standard oxaliplatin infusions). The primary endpoint of this study was overall survival (OS) in cases and controls (Kaplan-Meier method with log-rank test comparisons).

Results: OS was 23.7 months $(95 \% \mathrm{Cl}, 15.3-30.9)$ for the 67 cases who underwent 337 RDDs, while for controls $(n=143)$, 0 S was 34.5 months $(95 \% \mathrm{Cl}, 21.7-55.5)$. There were no significant differences between the groups $(\mathrm{HR}, 1.42 ; 95 \% \mathrm{Cl}, 0.93-2.17 ; P=.104)$. Conclusions: Survival outcomes of oxaliplatin-hypersensitive patients who received oxaliplatin via RDD did not differ significantly from those of control patients who received oxaliplatin via standard administration. Receiving oxaliplatin by means of RDD might be an effective therapeutic alternative for oxaliplatin-hypersensitive patients.
\end{abstract}

Key words: Drug allergy. Desensitization. Survival study. Hypersensitivity. Oxaliplatin. Skin test. Drug provocation test. Chemotherapy.

\section{Resumen}

Antecedentes: Las reacciones de hipersensibilidad al oxaliplatino podrían afectar al pronóstico vital cuando fuerzan a los pacientes a cambiar de tratamiento o cuando impiden que lo finalicen. La desensibilización rápida medicamentosa permite que estos pacientes reciban sus tratamientos de primera elección. Sin embargo, no existen datos sobre si la desensibilización rápida medicamentosa podría tener algún efecto sobre la eficacia del oxaliplatino.

Objetivo: El objetivo de este estudio es evaluar los efectos que la desensibilización rápida medicamentosa al oxalipatlino pudiera tener sobre la eficacia del tratamiento en los pacientes alérgicos al oxaliplatino sometidos a desensibilización.

Métodos: Estudio retrospectivo comparando datos de supervivencia, durante un periodo de 7 años, de pacientes alérgicos al oxaliplatino (recibiendo oxaliplatino mediante desensibilización rápida medicamentosa) y controles no alérgicos (recibiendo administraciones estándar de oxaliplatino). La supervivencia global se seleccionó como el criterio de valoración de la eficacia principal y se analizó con el estimador Kaplan-Meier utilizando comparaciones mediante la prueba de log-rank.

Resultados: La supervivencia global de los 67 casos fue de 23,7 meses (IC95\%, 15,3-30,9), que se sometieron a 337 desensibilizaciones rápidas medicamentosas. Para los 143 controles la supervivencia global fue 34,5 meses (IC95\%, 21,7-55,5). No se encontraron diferencias significativamente estadísticas entre ambos grupos ( $H R, 1,42 ; I C 95 \%, 0,93-2,17 ; P=0,104)$.

Conclusiones: Los resultados de supervivencia de los pacientes sometidos a desensibilización no fueron significativamente distintos a los de los controles que recibieron oxaliplatino de forma estándar. La desensibilización se presenta como una alternativa para recibir oxaliplatino de forma eficaz en pacientes alérgicos.

Palabras clave: Alergia a medicamentos. Desensibilización. Estudio de supervivencia. Hipersensibilidad. Oxaliplatino. Pruebas cutáneas. Provocación controlada. Quimioterapia. 


\section{Introduction}

Colorectal cancer is the third most common cancer in men and the second in women. The worldwide prevalence in 2012 was over 1300000 affected patients. About 20\% of cases are diagnosed at an advanced stage, with a relative 5-year survival rate of approximately $11 \%-12 \%[1,2]$. Standard treatment for these patients includes oxaliplatin-based chemotherapy regimens [3].

The incidence of drug hypersensitivity reactions (DHRs) to oxaliplatin has been increasing, and $21 \%-37 \%$ of cases are severe [4-6]. Because the reactions usually appear after a median of 8 uneventful administrations [4-6], they may jeopardize completion of the scheduled treatment sessions or force patients into unexpected changes in therapy when they are already successfully receiving standard oxaliplatin-based chemotherapy. Thus, DHRs to antineoplastic agents such as oxaliplatin may lead to first-choice drug avoidance, which could in turn negatively affect prognosis [4-16].

At Ramon y Cajal University Hospital (RCUH), Madrid, Spain, our group previously validated skin testing, specific IgE, and drug provocation testing (DPT) for the diagnosis of DHRs to oxaliplatin [6]. DPT involves the controlled administration of a drug to study DHRs and is considered the gold standard for establishing or excluding a diagnosis of drug hypersensitivity $[5,6,17]$. DPT is useful for ruling out a diagnosis of hypersensitivity in a considerable percentage of patients and therefore for preventing nonhypersensitive patients from unnecessary desensitizations [6].

The RCUH rapid drug desensitzation (RDD) protocol was recently validated in a large cohort study [5]. RDD is a therapeutic technique that enables drug-allergic patients to receive a drug they are hypersensitive to. It induces a temporary state of tolerance to a drug responsible for a proven DHR by means of the administration of progressively increasing doses of the culprit drug in a stepwise manner. This approach has been validated in vivo and in vitro [5,7-15]. Thus, RDD allows reactive patients to safely receive their first-choice treatments [5-15]. However, survival data on patients receiving oxaliplatin by means of RDD have not been reported.

The primary objective of this study was to evaluate the effect of RDD on the survival rates of oxaliplatinhypersensitive patients.

The secondary objective was to describe relevant data on the management of affected patients, with emphasis on the role of DPT and the efficacy and safety of RDD.

\section{Methods}

\section{Study Design}

We performed a retrospective analysis to compare survival data from oxaliplatin-hypersensitive patients receiving oxaliplatin by RDD and nonallergic controls receiving standard oxaliplatin infusions.

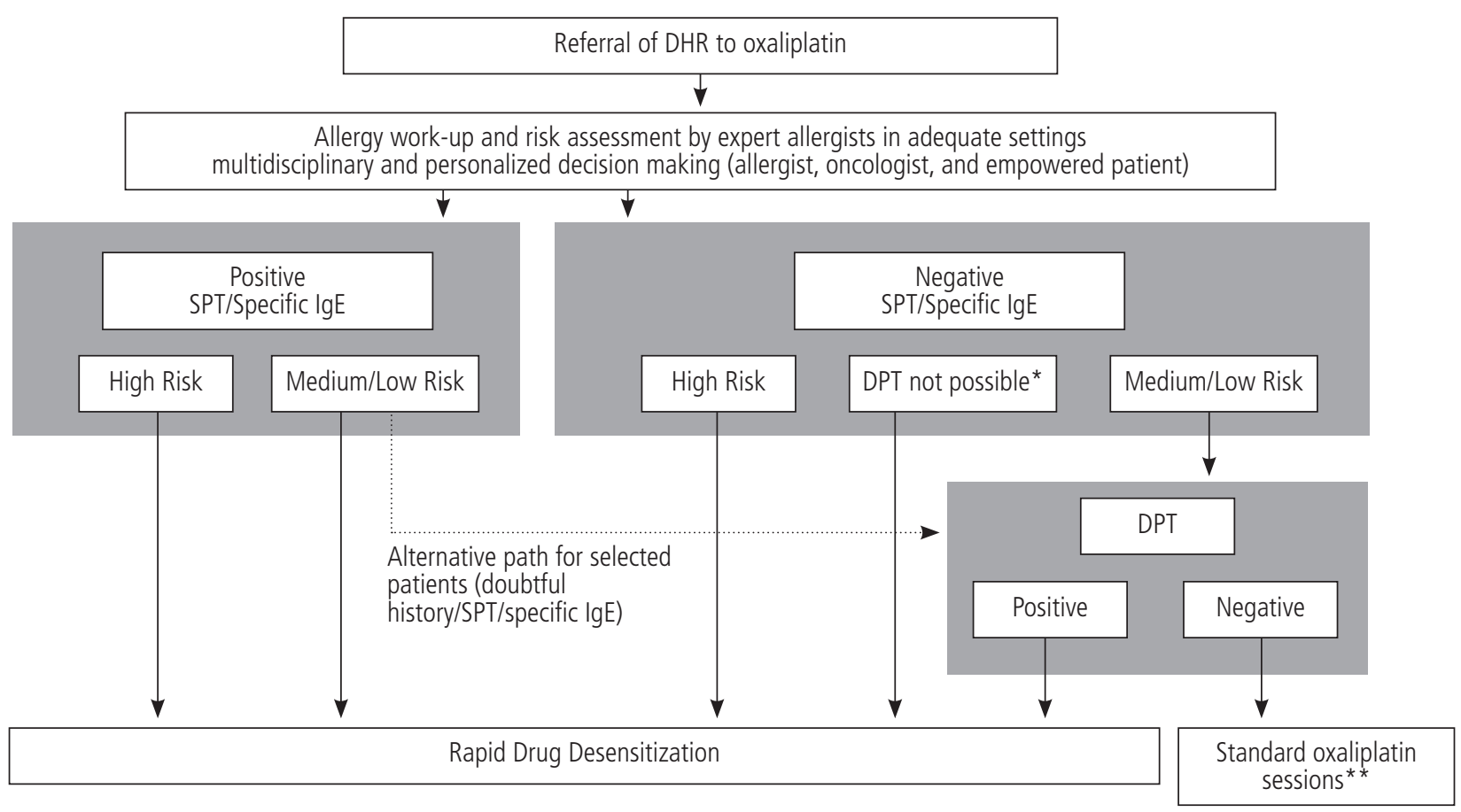

${ }^{*}$ DPT not possible (eg, inadequate installations, insufficient personnel, patient does not consent to DPT).

**Platin-reactive patients receiving standard sessions after negative DPTs might be in need of tailored follow-up including preventive screening ST, as 'positive converters' have been reported [6].

Figure 1. Flow chart for systematic allergy work-up of DHRs to oxaliplatin in the RCUH drug desensitization program. DHR indicates drug hypersensitivity reaction; DPT, drug provocation test; RCUH, Ramón y Cajal University Hospital; IgE, immunoglobulin E; RDD, rapid drug desensitization; SPT, skin prick testing. 


\section{Study Population}

The study population comprised patients with advanced colorectal cancer (stage IV) reacting to oxaliplatin and included in the patient cohort of the desensitization program at RCUH over a 7-year period (May 2009 to May 2016).

\section{Allergy Work-up}

As in previous studies by our group [5-12], all referred patients underwent a systematic allergy study based on clinical history, classification of severity according to Brown [18], skin testing (ST), blood testing (including oxaliplatin-specific IgE and tryptase), risk assessment, and drug provocation testing (DPT), when the appropriate criteria were met (Figure 1).

\section{Skin Testing and Specific IgE}

ST was performed following standard international methodology $[5-7,19,20]$ and in a dedicated space because of the risk of anaphylaxis [21]. The results were assessed according to international guidelines, as reported in previous

Table 1. RCUH Standard Rapid Drug Desensitization Protocol for a Total Dose of $200 \mathrm{mg}$ of Oxaliplatin Meant to be Infused in 2 Hours at $125 \mathrm{~mL} / \mathrm{h}$

\begin{tabular}{|c|c|c|c|c|c|c|c|}
\hline \multirow{4}{*}{$\begin{array}{l}\text { Total dose } \\
\text { Solution A } \\
\text { Solution B } \\
\text { Solution C }\end{array}$} & $200 \mathrm{mg}$ & \multicolumn{3}{|c|}{$\begin{array}{l}\text { Solution } \\
\text { Concentration }\end{array}$} & \multicolumn{2}{|c|}{$\begin{array}{l}\text { Total Dose in Each } \\
\text { Solution }(\mathrm{mg})\end{array}$} & Drug \\
\hline & $250 \mathrm{~mL}$ & & $0.016 \mathrm{mg} / \mathrm{mL}$ & & 4 & & Oxaliplatin \\
\hline & $250 \mathrm{~mL}$ & & $0.16 \mathrm{mg} / \mathrm{mL}$ & & 40 & & Oxaliplatin \\
\hline & $250 \mathrm{~mL}$ & & $0.8 \mathrm{mg} / \mathrm{mL}$ & & 200 & & Oxaliplatin \\
\hline Step & Solution & Rate, $\mathrm{mL} / \mathrm{h}$ & $\begin{array}{l}\text { Volume } \\
\text { administered, } \mathrm{mL}\end{array}$ & $\begin{array}{l}\text { Time, } \\
\text { min }\end{array}$ & $\begin{array}{l}\text { Dose } \\
\text { administered, } \\
\mathrm{mg}\end{array}$ & $\begin{array}{l}\text { Fold increase } \\
\text { per step, } \\
\mathrm{mg} / \mathrm{min}\end{array}$ & $\begin{array}{l}\text { Approximative } \\
\text { cumulative dose } \\
\text { infused, mg }\end{array}$ \\
\hline 1 & $\mathrm{~A}$ & 88 & 22 & 15 & 0.0 & NA & 0.0 \\
\hline 2 & A & 100 & 25 & 15 & 0.4 & NA & 0.4 \\
\hline 3 & $\mathrm{~A}$ & 200 & 50 & 15 & 0.8 & $\mathrm{x} 2$ & 1.2 \\
\hline 4 & A & 400 & 100 & 15 & 1.6 & $\mathrm{x} 2$ & 2.8 \\
\hline 5 & B & 88 & 22 & 15 & 0.0 & NA & 2.8 \\
\hline 6 & B & 100 & 25 & 15 & 4.0 & $\mathrm{x} 2.5$ & 6.8 \\
\hline 7 & B & 200 & 50 & 15 & 8.0 & $\mathrm{x} 2$ & 14.8 \\
\hline 8 & B & 400 & 100 & 15 & 16.0 & $\mathrm{x} 2$ & 30.8 \\
\hline 9 & $\mathrm{C}$ & 88 & 22 & 15 & 0.0 & NA & 30.8 \\
\hline 10 & $\mathrm{C}$ & 125 & 211.5 & 101.52 & 169.2 & $\mathrm{x} 1.6$ & 200.0 \\
\hline
\end{tabular}

Total infusion time: $236.52 \mathrm{~min}(3.9 \mathrm{~h})$.

Premedication: Ideally, premedication should comply with the manufacturer's instructions and institutional protocols for standard oxaliplatin infusion. We do not recommend additional premedication with corticosteroids or antihistamines as a measure to prevent breakthrough reactions [5,6,12]. However, tailored premedication may be added at the physician's discretion [5-12].

Total dose calculation and discarded volume: Not all the volume in solutions A, B, or C is infused. We start the protocol with solution $\mathrm{A}$, which contains a $1 / 50$ dilution, then solution $\mathrm{B}$ with a $1 / 5$ dilution, and we end with a final solution $\mathrm{C}$ containing a full concentration of the culprit drug. The total dose to be infused during solution $\mathrm{C}$ is calculated by subtracting the cumulative dose administered in steps 1-8 from the total desired dose.

Adjustments to the volume of the bags: The standard volume in the solution bags for the RCUH rapid drug desensitization protocol is $250 \mathrm{~mL}$. In some cases, bag volume may need to be adjusted depending on the manufacturer's instructions.

Additional bags for high-risk patients: Whenever needed in high-risk patients, additional solutions with lower concentrations than solution A may be added previously to solution A to ensure a more cautious starting dose, and, whenever possible, as determined on the basis of an endpoint titration according to local protocols (in the case of positive skin tests).

Flushing steps: Each solution uses an individual infusion line previously primed with $22 \mathrm{~mL}$ of the dilutor substance. Steps 1,5 , and 9 are considered "line-flushing steps" (in which $22 \mathrm{~mL}$ of the dilutor substance is administered).

Adjustments to the final infusion rate: Step 10 may be adapted to the desired final infusion rate according to the standard regimens indicated by the referring oncologist (additional steps may be added in order to reach higher infusion rates while maintaining a maximum dose increasing by 2 -fold to 2.5 -fold with each step).

Avoiding human errors: Infusion pumps with automatic multistep infusion options (Alaris SE 2-channel) were used to avoid human errors associated with manually changing infusion rates every 15 minutes. We used 22-mL infusion systems for these pumps (Alaris SE I Pump SmartSite Infusion Set).

Abbreviation: NA, not applicable; RCUH, Ramon y Cajal University Hospital. 
articles by our group [5-12,19]. The concentrations used for skin prick testing (SPT) were $5 \mathrm{mg} / \mathrm{mL}$ and $0.5 \mathrm{mg} / \mathrm{mL}$. For intradermal testing (IDT) we only considered the $0.5 \mathrm{mg} / \mathrm{mL}$ concentration, given that we found false-positive results when using $5 \mathrm{mg} / \mathrm{mL}$ for IDT [6].

Oxaliplatin-specific IgE was measured using ImmunoCAP (Thermo Fisher Scientific). Data from previous studies suggest that $0.35 \mathrm{kU} / \mathrm{L}$ is the ideal cut-off in our population [6].

\section{Drug Provocation Testing}

DPT is a helpful but risky technique that should only be performed by expert allergists in adequate settings after careful assessment of risks. DPT consisted of administering the next scheduled treatment with the culprit drug following the same detailed recommendations set out in recent articles by our group $[5,6]$. The standard criterion for a positive final diagnosis of hypersensitivity in this study was that an unequivocal clinical history was not considered sufficient [5-12]; patients also needed to have a positive ST and/or positive DPT and/or positive oxaliplatin-specific IgE.

The standard criterion for a negative diagnosis of hypersensitivity in this study was a negative DPT result.

Patients who did not meet the criteria for a positive or negative diagnosis were considered inconclusive.

\section{Rapid Drug Desensitization}

Since the tolerance induced by RDD is temporary, candidates received all subsequent oxaliplatin treatments by means of RDD. Our group previously validated an RDD protocol designed to last approximately 4 hours [5,7]. Table 1 presents a practical example of an RCUH/RDD protocol designed for 1 of the study patients and shows information on protocol design.

The inclusion criteria for being considered a candidate for RDD were as follows: (1) symptoms compatible with immediate type 1 DHRs during the drug infusion; (2) a firstchoice indication by the referring oncologist to be treated with the culprit drug; (3) signature of the informed consent document; and (4) a high risk or a positive final diagnosis of hypersensitivity to oxaliplatin.

\section{Selection of Patients for Survival Analysis}

Cases: In order to maintain homogeneity, only patients with advanced colorectal cancer were included in this study, according to the following inclusion criteria: (1) confirmed diagnosis of advanced colorectal cancer with objective and measurable oncological stage IV disease according to the American Joint Committee on Cancer Staging Manual [22]; (2) Eastern Cooperative Oncology Group performance status of $0-1$ [23]; (3) life expectancy greater than 12 weeks; (4) receiving oxaliplatin by means of RDD after experiencing a DHR to oxaliplatin; (5) signed informed consent.

Controls: All controls were selected from patients who were receiving treatment with oxaliplatin by means of standard infusions at our institution during the same period the cases were being treated and who met inclusion criteria (1), (2), (3), and (5).

We selected a group of unequivocally nonhypersensitive control patients. Nonreactive controls were retrospectively selected from the Medical Oncology Department databases and matched by sex, right-sided primary tumor location, grade of histological differentiation, therapeutic line, and KRAS mutation. We also included those patients from the Allergy Department cohort with a negative allergy work-up (hypersensitivity ruled out by negative ST and negative DPT, no previous RDD).

Given that some added controls (those added after a negative allergy work-up) are arguably "matched controls", we used logistic regression to compare characteristics between controls and cases and ensure adequate matching.

\section{Informed Consent Statement}

This study was approved by the Ethics Committee of RCUH, which validated the informed consent documents to be signed by the patient, allergist, and referring oncologist.

\section{Endpoints}

The primary endpoint was overall survival (OS, ie, the time from the beginning of oxaliplatin treatment/RDD to death from any cause). The secondary endpoint was progression-free survival (PFS, ie, the time from the beginning of oxaliplatin treatment/RDD to disease progression or death from any cause).

\section{Statistical Analysis}

The power calculation determined that at least 203 patients (patients plus controls) were needed to detect a clinically significant difference in survival between the groups ( $\mathrm{HR}, 0.67)$, with a $P$ value of .05 and a power of 0.8 . We set the case:control ratio at 1:2 to achieve the total sample size with the 67 patients selected. The survival analysis was based on univariate Cox proportional hazards regression, and a multivariate Cox regression model was run to assess prognostic variables. The potential prognostic variables that we selected were age, sex, primary tumor location, grade of differentiation, KRAS mutation, disease stage, primary tumor resection, surgery for metastatic disease, number of metastatic locations, therapeutic line, type of biologic treatment combined with chemotherapy, number of desensitization doses, best radiological response rate, and the number of therapeutic lines received after desensitization treatment. Survival analyses were performed between groups using Kaplan-Meier survival curves and a log-rank test. Analyses included all case patients who received at least 1 dose of oxaliplatin by means of RDD. All results with a $P$ value of $<.05$ were considered statistically significant. All analyses were carried out using the statistical package STATA, version 13.

In a retrospective analysis, we further investigated associations between tumor KRAS mutation status and oxaliplatin DHRs. The analyses included all case patients who received at least 1 dose of oxaliplatin by means of RDD.

\section{Results}

\section{Patient Characteristics}

During the 7-year study period, 102 patients with advanced colorectal cancer (stage IV) were referred to our desensitization program after a DHR to oxaliplatin.

Median age was 63 years (range, 21-85 years), and 54 (53\%) were men. In 18 patients (18\%), hypersensitivity 
to oxaliplatin was ruled out based on a negative DPT, and patients could continue receiving standard administrations of oxaliplatin (no need for RDD).

After completing the allergy work-up, only 67 patients $(66 \%)$ received oxaliplatin by means of RDD (65 had a positive diagnosis of hypersensitivity and 2 were inconclusive, according to our standard criteria). These 67 patients qualified as cases.

All initial reactions in cases occurred within 1 hour of administration of oxaliplatin and were consistent with immediate type 1 DHRs. No patients experienced nonimmediate DHRs. The initial reaction was moderate or severe (grade 2 or 3 ) in $40(60 \%)$, including 5 who experienced anaphylactic shock and cardiovascular collapse. The initial reaction was mild in 27 patients (40\%) (grade 1). Before the initial DHR, cases received a median of 11 uneventful oxaliplatin sessions

The characteristics of the 67 cases were very similar to those of the 102 referred patients: median age was 63 years (range, 22-85 years) and 38 (57\%) were men. The most common chemotherapy regimen was FOLFOX (leucovorin/5fluorouracil/oxaliplatin, 57 cases). Patients also received TOMOX (raltitrexed/oxaliplatin, 5 patients), XELOX (capecitabine/oxaliplatin, 1 patient), and other regimens. The location of the primary disease was left-sided colorectal
Table 2. Baseline Characteristics of the Study Population and Comparison by Logistic Regression

\begin{tabular}{lccc}
\hline Characteristics & $\begin{array}{c}\text { Cases } \\
(\mathrm{n}=67)\end{array}$ & $\begin{array}{c}\text { Controls } \\
(\mathrm{n}=143)\end{array}$ & $\begin{array}{c}P \\
\text { Value }\end{array}$ \\
\hline Mean age, y (range) & $63(22-85)$ & $70(18-97)$ & $<.05$ \\
Male/Female, \% & $38 / 29(57 / 43)$ & $89 / 54(62 / 38)$ & .29 \\
Primary site of disease, \% & & & \\
$\quad$ Right side & $16(24)$ & $37(26)$ & \\
$\quad$ Left side & $50(75)$ & $104(73)$ & .705 \\
KRAS mutation, \% & & & \\
$\quad$ Wild type & $28(42)$ & $36(25)$ & \\
$\quad$ Mutant & $30(45)$ & $39(27)$ & .269 \\
Histology, \% & & & \\
$\quad$ Well differentiated & $38(57)$ & $50(35)$ & \\
$\quad \begin{array}{l}\text { Moderately differentiated } \\
\text { Poorly differentiated }\end{array}$ & $20(30)$ & $65(45)$ & \\
& $4(6)$ & $7(5)$ & .802 \\
\hline
\end{tabular}

Regression Between Cases and Controls.

cancer in 50 cases $(75 \%)$, and $38(57 \%)$ had well differentiated histology.

Bevacizumab was administered in combination with oxaliplatin in $37(55 \%)$ cases. The $K R A S$ mutation was present

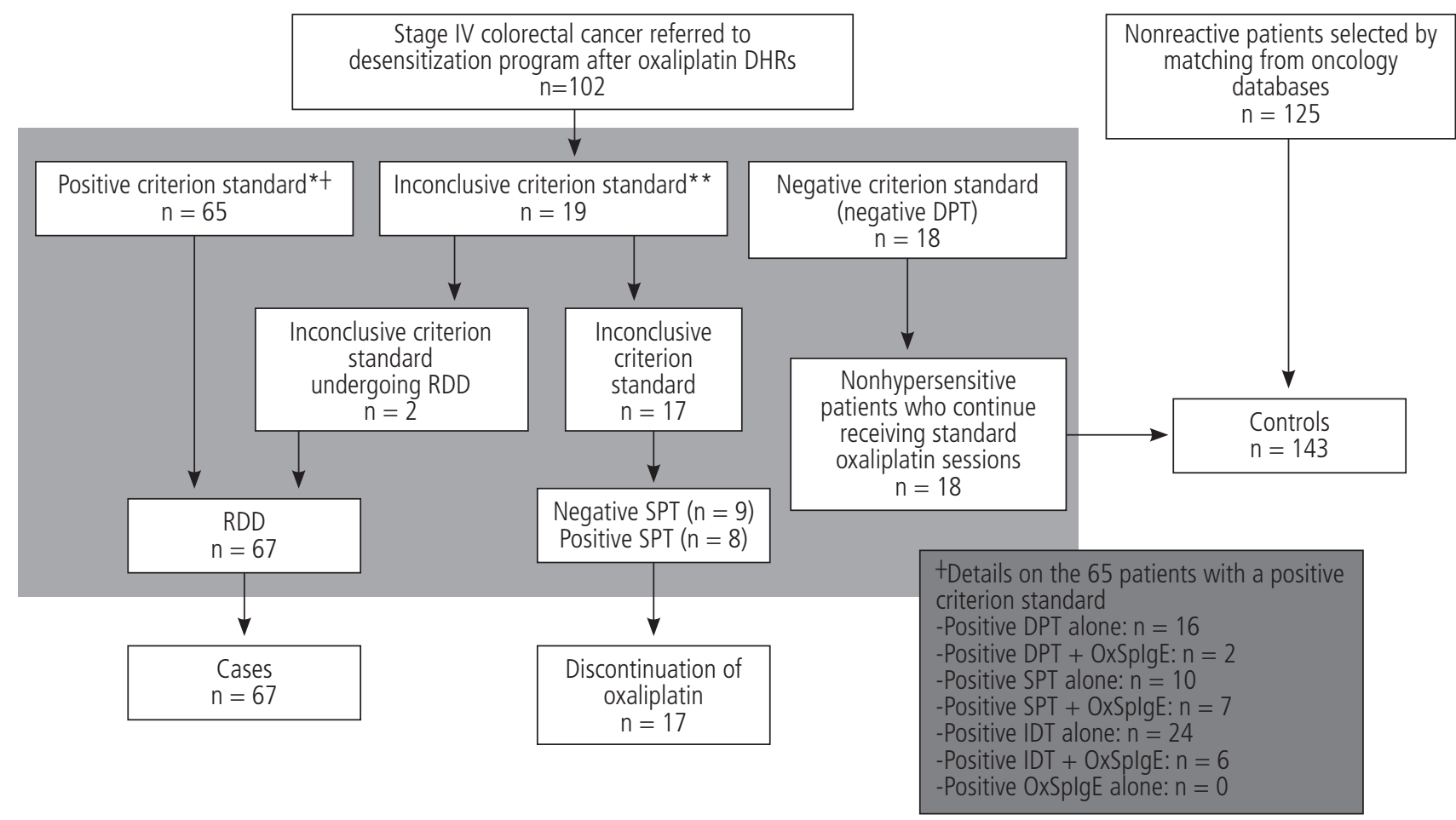

\footnotetext{
*Positive gold standard (unequivocal clinical history and positive skin testing and/or positive specific lgE and/or positive DPT).

**Inconclusive gold standard (patients who could not meet criteria for a positive or negative diagnosis). Some patients might be inconclusive because they discontinue oxaliplatin treatment after the initial DHR (for different reasons, namely, end of programmed treatment, do not consent, change to alternative therapy), thus making further study impossible.

Figure 2. Diagnostic flow chart and group assignment for the study population. DHR indicates drug hypersensitivity reaction; DPT, drug provocation test; IgE, immunoglobulin E; RDD, rapid drug desensitization; DPT, drug provocation testing; SPT, skin prick testing; IDT, intradermal testing; OxSplgE, oxaliplatin-specific lgE.
} 
in $30(45 \%)$. A total of 44 patients $(66 \%)$ had 2 or more objective radiological metastases, and only 7 (10\%) had not received surgical treatment for metastases.

Patient characteristics and the diagnostic flow chart are further described in Table 2 and Figures 1-2.

Seventeen of the 102 referred patients $(17 \%)$ changed to an alternative therapy or discontinued their treatments with oxaliplatin: 8 of these patients had a positive ST result, and 9 did not consent to DPT and/or RDD, despite a negative ST result and an initial mild reaction.

\section{Controls}

Eighteen of the 102 referred patients (18\%) had a negative allergy work-up (hypersensitivity ruled out by negative ST and negative DPT results and no previous RDD). These patients were added to the control group.

Additionally, 125 patients were selected as controls from the Oncology Department database and matched in order to ensure similar baseline characteristics.

The total number of controls was 143 patients.

\section{Rapid Drug Desensitization}

A total of 337 RDDs were performed in 67 patients. All RDDs were successful and all case patients could receive oxaliplatin by means of RDD. A total of 30 cases $(45 \%)$ underwent RDD on 6 occasions. Moreover, 34 (51\%) underwent RDD at least twice before any event (death or progression), and only 3 patients underwent RDD only once before death or progression. The largest number of administrations per patient was 25 . The safety profile was very good and similar to that reported in previous studies [5,7], with no breakthrough reactions in 320 RDDs $(95 \%)$. A total of 17 breakthrough reactions were observed, and most were mild (11/17); 4 were considered moderate and only 2 severe.

\section{Survival Analysis}

A total of 210 patients were included in the survival analysis (67 cases and 143 controls). The added controls are arguably 'matched controls', although we did not find any differences in any of the matched variables except for age. Median age was 63 years (ranging from 22 to 85 ) for cases $(n=67)$ and 70 for controls $(n=143)$. This difference was statistically significant (Table 2).

At the time of this analysis, 52 of the 67 cases (78\%) had progressive disease and $42(63 \%)$ had died. Median OS, defined as the time from the start of treatment to death from any cause or the time of the last follow-up visit, was 23.7 months (95\%CI, 15.3-30.9) for cases, while for controls it was 34.5 months (95\%CI, 21.7-55.5). Differences between groups were not statistically significant $(P=.1, \log$-rank; HR, $1.42 ; 95 \% \mathrm{CI}$, $0.93-2.17 ; P=.104)$. The results remained nonsignificant after adjusting for age $(P=.1, \log$-rank; HR, $1.5 ; 95 \% \mathrm{CI}, 0.97-2.36$; $P=.07)$. PFS was 8.8 months in the cases $(95 \% \mathrm{CI}, 7.9-12.8)$ and 13.04 months in the controls (95\%CI, 9.43-12.8); again, there were no significant differences between the groups, even after adjusting for age $(P=.1, \log$-rank; HR, $1.05 ; 95 \% \mathrm{CI}, 0.70$ $1.56 ; P=.83$ ) (Figure 3 ).

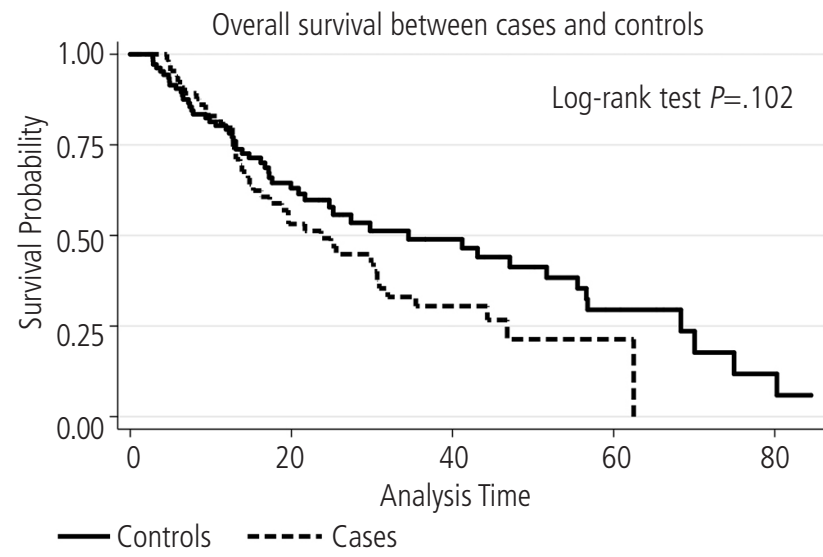

Figure 3. Curves for overall survival of 67 cases (RDD) and 143 controls (not RDD), defined as the time from the start of treatment to death from any cause or the time of the last follow-up visit. The median overall survival time was 23.7 months $(95 \% \mathrm{Cl}, 15.3-30.9)$ for cases and 34.5 months $(95 \% \mathrm{Cl}, 21.7-55.5)$ for controls. Comparison of log-rank curves between cases $(n=67)$ and controls $(n=143)$ revealed no significant differences between the groups ( $\mathrm{HR}, 1.42 ; 95 \% \mathrm{Cl}, 0.93-2.17 ; P=.104)$. RDD indicates rapid drug desensitization.

Some patients in both groups (cases and controls) might have undergone surgery at their initial diagnosis and may only have become patients with advanced colorectal cancer (stage IV) in a later phase after progression. These patients might be in need of chemotherapy a long time after their initial diagnosis. Therefore, we also show the data for OS defined as the time from the first diagnosis of cancer to death from any cause or the time of the last follow-up visit. OS was 61.6 months (95\%CI, 55.6-82.8) for cases $(\mathrm{n}=67)$, and 80.6 months (95\%CI, 77.1-not reached) for controls. Differences between groups were not significant $(P=.1$, log-rank; HR, 1.22; 95\%CI, $0.74-2.00 ; P=.431$ ) (Figure 4$)$.

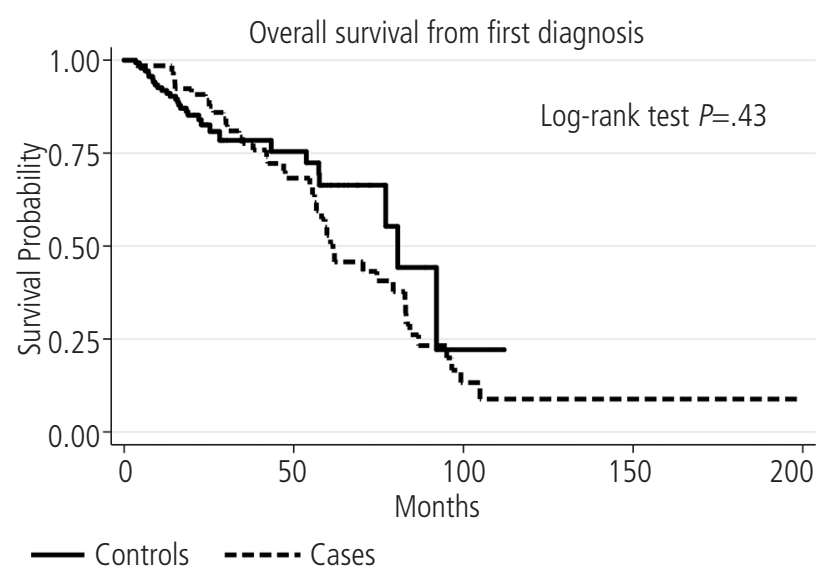

Figure 4. Curves for overall survival of 67 cases (RDD) and 143 controls (not RDD), defined as the time from diagnosis of cancer to death from any cause or the time of the last follow-up. The median overall survival time of cases was 61.6 months $(95 \% \mathrm{Cl}, 55.6-82.8)$, while for controls it was 80.6 months $(95 \% \mathrm{Cl}, 77.1$-not reached). Comparison of log-rank curves between cases and controls revealed no significant differences between the groups (HR, 1.22; $95 \% \mathrm{Cl}, 0.74-2.00 ; P=.431)$. 
Overall survival by line of treatment

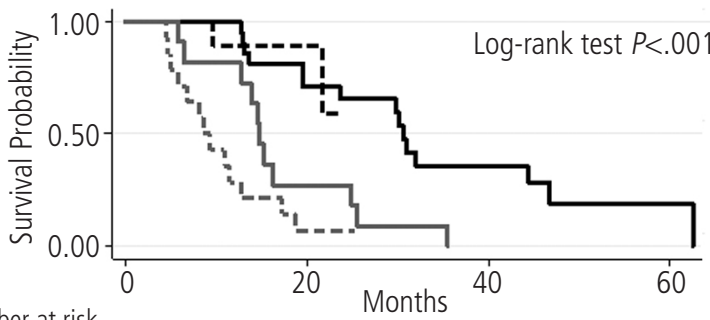

Number at risk

First-line 24

Second-line 10

Third-line 12

Further-line 14

-First - - - - Second

line

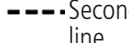

line
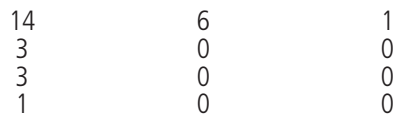

\begin{tabular}{|c|c|c|c|c|}
\hline $\begin{array}{l}\text { Line } \\
\text { of treatment }\end{array}$ & $\begin{array}{l}\text { Patients } \\
\text { (n) }\end{array}$ & $\begin{array}{l}\text { Median } \\
\text { (months) }\end{array}$ & \multicolumn{2}{|c|}{$\begin{array}{l}\text { 95\% Confidence } \\
\text { interval }\end{array}$} \\
\hline Adjuvant & 7 & $\begin{array}{c}\text { Not } \\
\text { achieved }\end{array}$ & $\begin{array}{c}\text { Not } \\
\text { achieved }\end{array}$ & $\begin{array}{c}\text { Not } \\
\text { achieved }\end{array}$ \\
\hline First-line & 24 & 30.65298 & 19.6468 & 46.7844 \\
\hline Second-line & 10 & $\begin{array}{c}\text { Not } \\
\text { achieved }\end{array}$ & 9.69199 & $\begin{array}{c}\text { Not } \\
\text { achieved }\end{array}$ \\
\hline Third-line & 12 & 14.81725 & 6.43942 & 24.9035 \\
\hline Further lines & 14 & 8.574948 & 4.99384 & 12.6817 \\
\hline All lines & 67 & 23.68789 & 15.2772 & 30.9158 \\
\hline
\end{tabular}

Figure 5. Curves for overall survival (defined as the time from the start of treatment to death from any cause or the time of the last follow-up visit) after stratification by line of treatment in the final 67 patients selected as cases (oxaliplatin-hypersensitive patients undergoing rapid drug desensitization).

Therapeutic options for advanced colorectal cancer are limited, and those available are administered consecutively when radiologic progression is observed. Thus, the therapy line refers to the running order of the administration of the different treatments [3]. Among the cases, 7 patients were treated postoperatively, 24 patients received a first-line treatment, 10 patients a second-line treatment, 12 patients a third-line treatment, and 14 patients further therapy lines (Figure 5). Survival times are usually different depending on the therapy line, and this was also true in the present study (Figure 5). Therefore, we analyzed and compared the data for OS (defined as the time from the start of treatment to death from any cause or the time of the last follow-up visit) between cases and controls after stratifying by the therapy line in which RDD was performed. The differences found were not statistically significant between the groups (Figure 6).

\section{Identifying Prognostic Factors for Survival in Cases}

The univariate analysis revealed the prognostic factors for poorer survival to be as follows: poor histological differentiation ( $\mathrm{HR}, 4.1 ; 95 \% \mathrm{CI}, 1.9-8.6 ; P<.001)$, stage IV disease at diagnosis (HR, $1.8 ; 95 \% \mathrm{CI}, 1.1-2.9 ; P=.02)$,
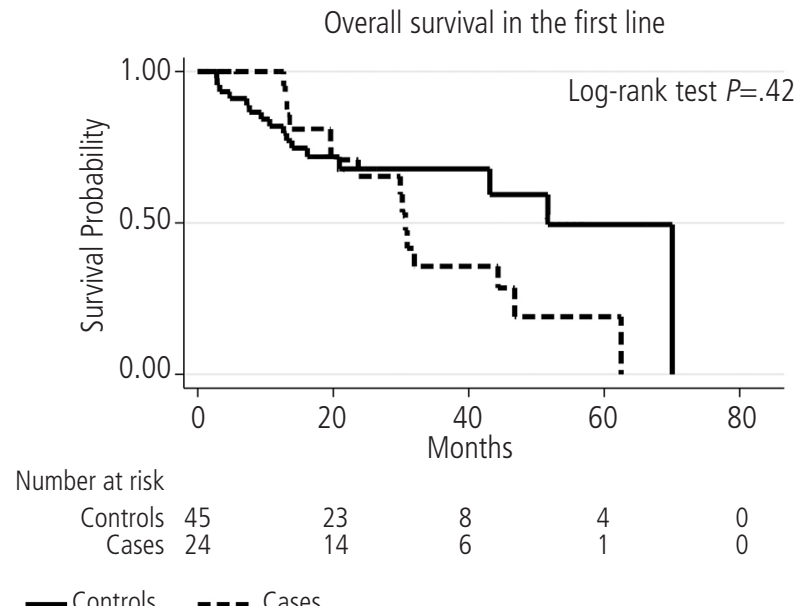

Controls - - - Cases

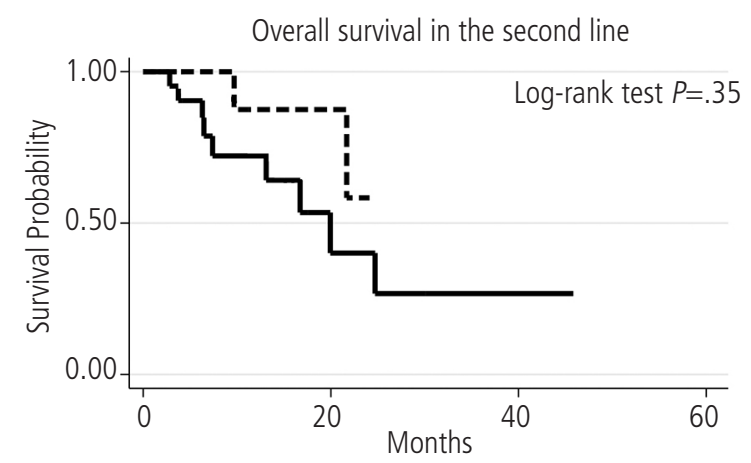

Number at risk

$\begin{array}{rlll}\text { Controls } 23 & 3 & 1 & 0 \\ \text { Cases } 9 & 3 & 0 & 0 \\ \text { Controls } & -\mathbf{- n} \text { Cases } & & \end{array}$

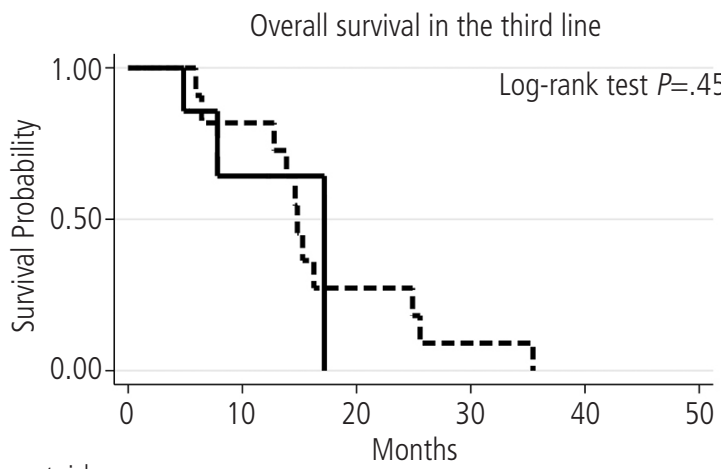

Number at risk

$\begin{array}{rrrrrrr}\text { Controls } & 8 & 3 & 0 & 0 & 0 & 0 \\ \text { Cases } & 12 & 9 & 3 & 1 & 0 & 0 \\ & & & & & & \end{array}$

Figure 6. Curves for overall survival (defined as the time from the start of treatment to death from any cause or the time of the last followup visit) after stratification by line of treatment and comparing cases with controls. 
Table 3. Cox Regression Analysis Including Age and the Prognostic Factors Identified in the Univariate Analysis

\begin{tabular}{lcccc} 
& HR & $P$ & \multicolumn{2}{c}{$95 \%$ CI } \\
\hline Cases & $2.86 \mathrm{e}+14$ & 1.000 & 0 & - \\
Age & 1.01 & .715 & 0.97 & 1.04 \\
Poorly differentiated & 2.84 & .152 & 0.68 & 11.93 \\
Stage IV at diagnosis & 3.56 & .01 & 1.35 & 9.41 \\
$\begin{array}{l}\text { Number of metastatic } \\
\text { locations }\end{array}$ & 0.74 & .166 & 0.48 & 1.13 \\
$\begin{array}{l}\text { Line in which RDD } \\
\text { is performed }\end{array}$ & 2.03 & $<.001$ & 1.51 & 2.73 \\
Bevacizumab treatment & 2.13 & .092 & 0.88 & 5.15 \\
\hline
\end{tabular}

Abbreviation: $\mathrm{RDD}$, rapid drug desensitization.

number of metastatic locations (HR, 1.4; 95\%CI, 1.1-1.7; $P=.001)$, therapy line in which RDD was performed (HR, 1.9; 95\%CI, 1.6-2.4; $P<.001)$, and treatment with bevacizumab combinations (HR, 3.1; 95\%CI, 1.5-6.5; $P=.002)$. In the multivariate Cox regression analysis including these factors and age, the presence of stage IV disease at diagnosis and the administration of RDD in later lines of treatment were the only significant and independent factors indicating poorer survival (Table 3).

Additionally, there was no association between the KRAS mutation and presenting with a DHR or between the KRAS mutation and severity of the DHR.

\section{Discussion}

\section{Primary and Secondary Endpoints}

We found no statistically significant differences in oxaliplatin efficacy outcomes between cases (oxaliplatinhypersensitive patients receiving oxaliplatin by means of RDD) and controls (patients receiving standard oxaliplatin infusions). The results were equally effective for both groups in terms of OS and in the subanalysis in terms of OS and PFS.

The survival outcomes and expected patterns (such as differences in survival rates depending on therapeutic line) for the cases we report were similar to those of the cases in previous survival analyses [24-26]. For example, while our median OS for third-line patients was 14.8 months, the median OS was 6.4 months and 7.1 months, respectively, in the classic CORRECT [26] and RECOURSE [25] trials. Regarding first-line patients, we obtained a median OS of 30.7 months, which is similar to the findings in the classic FIRE-3 trials [24], where the median OS was 28.7 months in the FOLFIRI/cetuximab group and 25.0 months in the FOLFIRI/bevacizumab group.

These data show that RDD does not seem to affect the efficacy of oxaliplatin when compared with standard infusions; therefore, receiving oxaliplatin by means of RDD could be an effective therapeutic alternative for oxaliplatin-hypersensitive patients (allowing for a median OS of 30.7 months in firstline treatments in the RDD population we report). Moreover, oxaliplatin shows better survival rates when it is administered in first-line treatments; this pattern is also observed when it is administered by means of RDD. Therefore, RDD should become a key therapeutic technique to be considered in oxaliplatin-hypersensitive patients and should be available as a reasonable option in first-line treatments, not only as a late alternative for further lines.

Consistent with our findings, although with a different drug, Sloane et al [14] showed that the efficacy of carboplatin was not reduced in allergic patients and that RDD protocols are as effective as regular infusions.

\section{The Role of DPT}

In a previous study, we showed that up to $32 \%$ of platinreactive patients may be nonhypersensitive (negative DPT) and, therefore, can avoid RDD [6]. Interestingly, a negative DPT was observed only in the specific $18 \%$ of the population of this study with a negative work-up, which could potentially be linked to patients with advanced colorectal cancer being exposed to a larger number of oxaliplatin sessions and therefore a higher risk of allergic sensitization.

Previous publications [5-16] discuss the unique role of the allergist for effectively and safely assessing patients with DHRs to chemotherapy. An adequate allergy work-up helps reactive patients receive their first-choice treatment and avoids unnecessary RDDs. In the present study, $83 \%$ of patients could benefit directly from an allergy work-up and continue with oxaliplatin (either after a negative DPT or by means of RDD), which might have been otherwise discontinued. Implementation of DPT helped 18 patients (18\%) in the present study to continue with standard oxaliplatin infusions after ruling out hypersensitivity to oxaliplatin (negative DPT). Additionally, systematic expert assessment will identify those patients who are more vulnerable to the nonzero risk of anaphylaxis during RDD [5].

\section{The Role of RDD}

Even if more studies are needed for different drugs, we recommend RDD as a therapeutic option for all cancer patients who present with DHRs to their first-choice treatment. Our data highlight key features of RDD that have been addressed elsewhere [6-15]. RDD is an efficacious, cost-effective, and safe therapeutic option, even in patients who present with severe DHRs, provided that adequate facilities are available (eg, rapid access to the intensive care unit, dedicated allergy technical areas with specific risk management procedures, the physical presence of an allergist at the bedside, an appropriate nurse:patient ratio, monitoring, crash cart). In addition, patients must be appropriately assessed by expert allergists leading a multidisciplinary team of trained personnel (eg, pharmacists, nurses, medical intensive care unit personnel) in close collaboration with referring oncologists [5].

\section{Limitations}

Our study is limited by the fact that it was performed at a single center. In addition, it is subject to the following issues with the control group regarding power calculations and matching. The initial analysis was not powered for evaluating 
the subanalysis of OS in the different treatment lines, thus making the results of this subanalysis by line of treatment prone to false-negative (noninferior) results. Furthermore, given the differences in age, the comparator arm is not a complete group of matched controls (18 controls were added after a negative allergy work-up); however, specific statistical corrections for this were applied where necessary.

\section{Conclusions}

This is the first reported survival study in oxaliplatinhypersensitive patients undergoing RDD. Data were obtained from one of the largest reported series of oxaliplatin RDDs in patients with advanced colorectal cancer, comprising 337 procedures in 67 patients over a 7 -year period. In order to avoid selection bias, the diagnosis of hypersensitivity to oxaliplatin in this cohort was only confirmed after a strict work-up including DPT. According to our results, RDD does not seem to affect the efficacy of oxaliplatin based on survival rates when compared with standard infusions, even when we stratify by therapeutic line. Therefore, receiving oxaliplatin by means of RDD could be an effective therapeutic alternative for oxaliplatin-hypersensitive patients and should be available as a reasonable option in first-line treatments, not only as a late alternative for further lines.

\section{Acknowledgments}

We would like to thank all the nurses from our team and all participants in the multidisciplinary Desensitization Program of the Ramon y Cajal University Hospital Allergy Division, especially members from the Pharmacy Department, Clinical Oncology Department, and Medical Intensive Care Unit. Above all, we are grateful to the patients for their active and enthusiastic collaboration.

\section{Funding}

The authors declare that no funding was received for the present study.

\section{Conflicts of Interest}

The authors declare that they have no conflicts of interest.

\section{References}

1. Ferlay J, Soerjomataram I, Ervik M, Dikshit R, Eser S, Mathers C, et al. GLOBOCAN 2012 v1.0, Cancer Incidence and Mortality Worldwide: IARC CancerBase No. 11 [Internet]. Lyon, France: International Agency for Research on Cancer. 2013 [Accessed on 19/10/2016]. Available from: http://globocan.iarc.fr.

2. Howlader N, Noone AM, Krapcho M, Garshell J, Miller D, Altekruse SF, et al. SEER Cancer Statistics Review, 1975-2014, National Cancer Institute [Internet]. Bethesda (MD): National Cancer Institute (US). 2014 [update: April 2017; Accessed on 29/04/2017]. Available from: https://seer.cancer.gov/ csr/1975_2014/.
3. Van Cutsem E, Nordlinger B, Cervantes A. Advanced colorectal cancer: ESMO Clinical Practice Guidelines for treatment. Ann Oncol [Internet]. 2010 [accessed on 17/10/2016];(Suppl 5):v93-v97.

4. Polyzos A, Tsavaris N, Gogas H, Souglakos J, Vambakas L, Vardakas $\mathrm{N}$, et al. Clinical features of hypersensitivity reactions to oxaliplatin: A 10-Year Experience. Oncology. 2009;76:3641.

5. Madrigal-Burgaleta R, Bernal-Rubio L, Berges-Gimeno MP, Carpio-Escalona LV, Gehlhaar P, Alvarez-Cuesta E. A large single hospital experience using drug provocation testing and rapid drug desensitization in hypersensitivity to antineoplastic and biological agents. J Allergy Clin Immunol Pract. 2019;7:618-32.

6. Alvarez-Cuesta $E$, Madrigal-Burgaleta $R$, Angel-Pereira $D$, Ureña-Tavera A, Zamora-Verduga M, Lopez-Gonzalez $\mathrm{P}$, et al. Delving into cornerstones of hypersensitivity to antineoplastic and biological agents: Value of diagnostic tools prior to desensitization. Allergy. 2015;70:784-94.

7. Madrigal-Burgaleta R, Berges-Gimeno MP, Angel-Pereira $D_{\text {, }}$ Ferreiro-Monteagudo R, Guillen-Ponce C, Alvarez-Cuesta E, et al. Hypersensitivity and desensitization to antineoplastic agents: Outcomes of 189 procedures with a new short protocol and novel diagnostic tools assessment. Allergy. 2013;68:853-61.

8. Ureña-Tavera A, Zamora-Verduga $M$, Madrigal-Burgaleta $\mathrm{R}$, Angel-Pereira D, Berges-Gimeno MP, Alvarez-Cuesta E. Hypersensitivity reactions to racemic calcium folinate (leucovorin) during FOLFOX and FOLFIRI chemotherapy administrations. J Allergy Clin Immunol. 2015;135:1066-7.

9. Madrigal-Burgaleta R, Berges-Gimeno MP, Angel-Pereira D, Guillén-Ponce C, Sanz ML, Alvarez-Cuesta E. Desensitizing oxaliplatin-induced fever: a case report. J Investig Allergol Clin Immunol. 2013:23:435-6.

10. Alvarez-Cuesta E, Madrigal-Burgaleta R, Berges-Gimeno MP Angel-Pereira D. Rapid desensitization to chemotherapy and monoclonal antibodies is effective and safe. Reply. Allergy. 2013;68(11):1483-4.

11. Lopez-Gonzalez P, Madrigal-Burgaleta R, Carpio-Escalona $\mathrm{LV}$, Bernal-Rubio L, Berges-Gimeno MP, Alvarez-Cuesta E. Assessment of antihistamines and corticosteroids as premedication in rapid drug desensitization to paclitaxel: outcomes in 155 procedures. J Allergy Clin Immunol Pract. 2018:6:1356-62.

12. Solano-Solares $E_{1}$ Madrigal-Burgaleta $R$, Carpio-Escalona LV, Bernal-Rubio L, Berges-Gimeno MP, Alvarez-Cuesta E. Chemotherapy in Mastocytosis: Administration Issues, Hypersensitivity, and Rapid Drug Desensitization. J Investig Allergol Clin Immunol. 2017;27:315-7.

13. Caiado J, Castells M. Presentation and Diagnosis of Hypersensitivity to Platinum Drugs. Curr Allergy Asthma Rep. 2015:15:15.

14. Sloane D, Govindarajulu U, Harrow-Mortelliti J, Barry W, Hsu Fl, Hong D, et al. Safety, Costs, and Efficacy of Rapid Drug Desensitization to Chemotherapy and Monoclonal Antibodies. J Allergy Clin Immunol Pract. 2016;4:497-504.

15. Bonamichi-Santos R, Castells M. Desensitization for Drug Hypersensitivity to Chemotherapy and Monoclonal Antibodies. Curr Pharm Des. 2016;22:6870-80. 
16. Ichikawa Y, Goto A, Hirokawa S, Kijima M, Ishikawa T, Chishima $T$, et al. Allergic reactions to oxaliplatin in a single institute in Japan. Jpn J Clin Oncol. 2009;39:616-20.

17. Aberer W, Kränke B. Provocation tests in drug hypersensitivity. Immunol Allergy Clin North Am. 2009;29:567-84.

18. Brown SG. Clinical features and severity grading of anaphylaxis. J Allergy Clin Immunol. 2004;114:371-6.

19. Brockow K, Romano A, Blanca M, Ring J, Pichler W, Demoly $P$. General considerations for skin test procedures in the diagnosis of drug hypersensitivity. Allergy. 2002;57:45-51.

20. Limsuwan T, Castells MC. Outcomes and safety of rapid desensitization for chemotherapy hypersensitivity. Expert Opin Drug Saf. 2010;9:39-53.

21. Martin-Lazaro J, Firvida JL, Berges-Gimeno P. Anaphylaxis after oxaliplatin allergy skin testing. J Investig Allergol Clin Immunol. 2014;24:269-70.

22. Amin MB, Edge S, Greene F, Byrd DR, Brookland RK, Washington MK, et al. AJCC Cancer Staging Manual [Internet]. 7th Edition. New York: Springer-Verlag, [last update: April 2017; accessed on: 19/10/2016]. Available at: https://cancerstaging.org/references-tools/deskreferences/ Documents/AJCC6thEdCancerStagingManualPart1.pdf

23. Oken MM, Creech RH, Tormey DC, Horton J, Davis TE, McFadden ET, et al. Toxicity and response criteria of the Eastern Cooperative Oncology Group. Am J Clin Oncol. 1982;5:64955.

24. Heinemann $V$, von Weikersthal LF, Decker T, Kiani A, VehlingKaiser U, Al-Batran SE, et al. FOLFIRI plus cetuximab versus FOLFIRI plus bevacizumab as first-line treatment for patients with metastatic colorectal cancer (FIRE-3): a randomised, open-label, phase 3 trial. Lancet Oncol. 2014;15:1065-75.

25. Mayer RJ, Van Cutsem E, Falcone A, Garcia-Carbonero R, Mizunuma N, Yamazaki K, et al; RECOURSE Study Group. Randomized trial of TAS-102 for refractory metastatic colorectal cancer. N Engl J Med. 2015;372:1909-19.

26. Grothey A, Van Cutsem E, Sobrero A, Siena S, Falcone A, Ychou $M$, et al. Regorafenib monotherapy for previously treated metastatic colorectal cancer (CORRECT): an international, multicentre, randomised, placebo-controlled, phase 3 trial. CORRECT Study Group. Lancet. 2013;381:303-12.

27. Lee MS, Menter DG, Kopetz S. Right Versus Left Colon Cancer Biology: Integrating the Consensus Molecular Subtypes. J Natl Compr Canc Netw. 2017; 15:411-9.

\footnotetext{
Manuscript received November 14, 2018; accepted for publication June 10, 2019.
}

\section{- Ricardo Madrigal-Burgaleta}

\author{
Hospital Universitario Ramon y Cajal \\ Servicio de Alergia \\ Ctra Colmenar Viejo, km 9,100 \\ 28034 Madrid, Spain \\ E-mail: ricardo.madrigal.md.phd@gmail.com
}

